\title{
Illuminating the molecular basis of diabetic arteriopathy: A proteomic comparison of aortic tissue from diabetic and healthy rats
}

\author{
Mia Jüllig,2*, Xiuyin Chen ${ }^{1 *}$, Martin J. Middleditch ${ }^{1,2}$, Greeshma Vazhoor $^{1}$, \\ Anthony J. Hickey ${ }^{1}$, Deming Gong ${ }^{1}$, Jun Lu ${ }^{1,3}$, Shaoping Zhang ${ }^{1,2}$, Anthony R. J. Phillips ${ }^{1,2,4}$ \\ and Garth J. S. Cooper ${ }^{1,2,5,6}$ \\ ${ }^{1}$ School of Biological Sciences, University of Auckland, Auckland, New Zealand \\ ${ }^{2}$ Maurice Wilkins Centre for Molecular Biodiscovery, Faculty of Science, University of Auckland, Auckland, New \\ Zealand \\ ${ }^{3}$ Department of Interdisciplinary Studies, Faculty of Health and Environmental Sciences, Auckland University of \\ Technology, Auckland, New Zealand \\ ${ }^{4}$ Department of Surgery, Faculty of Medical and Health Sciences, University of Auckland, Auckland, New Zealand \\ ${ }^{5}$ Department of Pharmacology, Medical Sciences Division, University of Oxford, Oxford, UK \\ ${ }^{6}$ Centre for Advanced Discovery and Experimental Therapeutics, NIHR Manchester Biomedical Research Centre, \\ the University of Manchester, Manchester, UK
}

\begin{abstract}
Arterial disease is a major diabetic complication, yet the component molecular mechanisms of diabetic arteriopathy remain poorly understood. In order to identify major proteins/ pathways implicated in diabetic arteriopathy, we studied the effect of 16-wk untreated streptozotocin-induced diabetes on the rat aortic proteome. Specific protein levels in isolated aortas were compared in six discrete, pair-wise (streptozotocin-diabetic and non-diabetic agematched controls) experiments in which individual proteins were identified and quantified by iTRAQ combined with LC-MS/MS. A total of 398 unique non-redundant proteins were identified in at least one experiment and 208 were detected in three or more. Between-group comparisons revealed significant changes or trends towards changes in relative abundance of 51 proteins (25 increased, 26 decreased). Differences in levels of selected proteins were supported by Western blotting and/or enzyme assays. The most prominent diabetesassociated changes were in groups of proteins linked to oxidative stress responses and the structure/function of myofibrils and microfilaments. Indexes of mitochondrial content were measurably lower in aortic tissue from diabetic animals. Functional cluster analysis also showed decreased levels of glycolytic enzymes and mitochondrial electron transport systemcomplex components. These findings newly implicate several proteins/functional pathways in the pathogenesis of arteriosclerosis/diabetic arteriopathy.
\end{abstract}

Received: April 26, 2010

Revised: June 23, 2010

Accepted: July 1, 2010

\section{Keywords:}

Aorta / Arterial mitochondria / Arterial remodelling / Biomedicine / Diabetic arteriopathy / Streptozotocin-diabetic rat

\footnotetext{
Correspondence: Professor Garth J. S. Cooper, Level 4, Thomas Building, School of Biological Sciences, University of Auckland, Private Bag 92019, Auckland, 1142 New Zealand

E-mail: g.cooper@auckland.ac.nz

Fax: +64-9-373-7045
}

\begin{abstract}
Abbreviations: CA, carbonic anhydrase; ECM, extracellular matrix; ETS, electron transport system; IPI, international protien index; mt, mitochondrial; MudPIT, multidimensional protein identification technology; SSAO, semicarbazide-sensitive amine oxidase; STZ, streptozotocin
\end{abstract}

\section{Introduction}

There is a close relationship between chronic hyperglycaemia and the onset/progression of the vascular complications of diabetes [1]. In diabetes, the arterial wall often becomes diffusely damaged by a pathogenic process termed diabetic arteriopathy, which is mediated via pathways/mechanisms

\footnotetext{
*These authors contributed equally to this study.
} 
that modify its structure/function, and which include, inter alia: glycation of extracellular proteins, diffuse fibrosis of the tunica media, structural rearrangement and compositional changes of the proteins/proteoglycans that comprise the extracellular matrix (ECM) and alterations in the cellular components (collectively termed 'remodelling'), and calcification. These processes combine to cause a pathological state named 'arteriosclerosis' that damages large and medium-sized arteries, which display increased stiffness, hardness, loss of elasticity and diffuse wall thickening [2]. Arteriosclerosis typically leads to or causes widespread structural/functional impairment of arteries throughout the arterial tree and ultimately results in progressive impairment of the circulation of the blood [3].

Arteriosclerosis is to be contrasted with the closely related but distinct pathogenic process termed 'atherosclerosis', which often accompanies it but which in its earlier stages is typically confined mainly to the tunica intima. Atherosclerosis comprises progressive focal lesions that initially present as discrete intimal thickenings with extracellular accumulation of lipid and modified proteoglycans, with subsequent infiltration of lipid-laden foamy macrophages. Atherosclerosis is also distinguished from arteriosclerosis by the distribution of atherosclerotic lesions [4], which is typically referred to as focal or, as it becomes more extensive, focal diffuse, as opposed to the diffuse nature of arteriosclerosis.

The specific proteins/molecular pathways damaged by diabetic arteriopathy remain poorly understood, and improved knowledge thereof is a prerequisite for improved understanding of arteriosclerosis, and new and improved therapeutic approaches that might ameliorate or reverse its impact in diabetic patients.

The ECM is in a dynamic flux of continual synthesis, degradation and reorganization of its components. Early disruption of ECM equilibrium probably contributes to increased aortic stiffness, and may explain why diabetic patients are at high risk of developing arterial complications such as atherosclerosis. Chronic hyperglycaemia appears to progressively impair the elastic properties of the aorta in diabetic individuals [5]. However, the precise molecular bases of the pathogenic mechanisms that underlie arteriosclerosis in diabetes have remained poorly characterized.

In order to better understand pathogenic processes that occur in the arteries of diabetic rats, we undertook a proteomic study of aortic tissue in rats with streptozotocin (STZ)-induced diabetes, a widely used animal model of the diabetic complications. STZ-diabetic rats display many of the hallmarks of diabetes-evoked vascular pathology, including hardness, stiffness and thickening of the aortic wall [6].

We have previously shown that aortic tissue from STZdiabetic rats exhibits hardness and stiffness upon palpation, consistent with that observed in patients with diabetic arteriosclerosis [7]. Here, we employed a gel-free multidimensional protein identification technology (MudPIT)/ iTRAQ approach, which enables the detection and quantitation of proteins, including those unsuitable for separation by two-dimensional gel electrophoresis due to factors, for example: extremes of hydrophobicity, molecular weight or pI, or insolubility.

To our knowledge, this is the first reported application of iTRAQ/MudPIT proteomics for the discovery of aortic proteins with altered expression in diabetes-associated arteriosclerosis.

\section{Materials and methods}

\subsection{Animals and tissue collection}

These studies were approved by institutional ethics and regulatory committees. Male Wistar rats $(220-250 \mathrm{~g})$ were assigned randomly to diabetic and non-diabetic control groups, injected intravenously with a single dose of STZ (Sigma-Aldrich, St Louis, MO, USA; $55 \mathrm{mg} / \mathrm{kg}$ body weight, in saline) or $0.5 \mathrm{~mL}$ saline vehicle only (diabetic and control animals, respectively). All animals were kept under a 12h:12-h light-dark cycle, at $21-23^{\circ} \mathrm{C}$ and $50 \pm 10 \%$ relative humidity, and maintained with standard rat chow and tap water ad libitum. Blood glucose levels and bodyweights were measured weekly throughout the study. At the end of the 16wk experimental period, the rats were anesthetized with halothane $(2 \% \mathrm{v} / \mathrm{v})$ in oxygen. The descending aorta was excised and washed thoroughly in PBS. For histology, tissue was immersed in optimal cutting temperature compound (Sakura Finetek, Torrance, CA, USA). Aortic tissue for proteomic study was snap-frozen in liquid nitrogen and stored at $-80^{\circ} \mathrm{C}$ until analyzed.

\subsection{Histology}

Sections $(5 \mu \mathrm{m}$ thick) were stained with Movat's pentachrome [8] and examined by light microscopy.

\subsection{Sample preparation for iTRAQ}

Aortic tissue ( $250 \mathrm{mg}$ ) from each of six animals per group was ground into fine powder (liquid $\mathrm{N}_{2}$ /agate mortar and pestle; Global Science, Auckland, NZ), and then supplemented with $400 \mu \mathrm{L}$ extraction buffer $(7 \mathrm{~mol} / \mathrm{L}$ urea, $2 \mathrm{~mol} / \mathrm{L}$ thiourea, $0.8 \%$ Surfact-Amps ${ }^{\circledR}$ X-100 (Thermo Scientific, New Zealand), $10 \mathrm{mmol} / \mathrm{L}$ DTT, $5 \mathrm{mmol} / \mathrm{L}$ EDTA, $10 \% \mathrm{v} / \mathrm{v}$ glycerol, $8 \mu \mathrm{mol} / \mathrm{L}$ pepstatin A and $16 \mu \mathrm{mol} / \mathrm{L}$ bestatin hydrochloride in $15 \mathrm{mM}$ phosphate buffer, $\mathrm{pH}$ 8.0). Extracts were incubated at room temperature for $1 \mathrm{~h}$ and sonicated (Vibra Cell ${ }^{\mathrm{TM}}$; Newton, CT, USA) for $5 \mathrm{~s}$. Following centrifugation at $16000 \times \mathrm{g}$ for $20 \mathrm{~min}$ at $4{ }^{\circ} \mathrm{C}$, supernatants were transferred to fresh microcentrifugefuge tubes. Samples were then alkylated by incubation with $50 \mathrm{mmol} / \mathrm{L}$ iodoacetamide (Bio-Rad, Hercules, CA, USA) for $2 \mathrm{~h}$ in the dark. Sample protein concentrations were determined (2D Quant; GE Healthcare, UK) and adjusted to 
$1 \mathrm{mg} / \mathrm{mL}$ with $15 \mathrm{mmol} / \mathrm{L}$ phosphate buffer, $\mathrm{pH} 8.0$, and $50 \mu \mathrm{g}$ of protein was digested with $2 \mu \mathrm{L}$ trypsin $(1 \mathrm{mg} / \mathrm{mL})$ (Promega, Madison, WI, USA) at $37^{\circ} \mathrm{C}$ for $4 \mathrm{~h}$. The resulting tryptic digests were desalted and dried using a vacuum concentrator (UVS400A; ThermoFisher, USA). Dried digests were resolubilized with $30 \mu \mathrm{L}$ dissolution buffer and labeled with iTRAQ 4-plex reagents (Applied Biosystems, Foster City, CA, USA) following the manufacturer's instructions. Equal volumes of labeled material from diabetic and control groups were combined and concentrated to approximately $30 \mu \mathrm{L}$, acidified with $10 \% \mathrm{v} / \mathrm{v}$ formic acid and diluted to $250 \mu \mathrm{L}$ with water (18 M $\Omega / \mathrm{cm}$ resistivity, Milli Q; Millipore Billerica, MA, USA).

\subsection{LC-MS/MS}

Samples were fractionated using ten $\mathrm{KCl}$ salt steps on a BioSCX II $0.3 \times 35$ mm column (Agilent Technologies, Santa Clara, CA, USA), and peptides were captured on a $0.3 \times 5$ mm C18 PepMap cartridge (LC Packings, Sunnyvale, CA, USA) followed by separation on a C18 300 SB $0.3 \times 100 \mathrm{~mm}$ Zorbax column (Agilent Technologies). A binary LC gradient was delivered at $6 \mu \mathrm{L} / \mathrm{min}$ as follows: 0-7 min 10\%B; $80 \min 35 \% \mathrm{~B}$; $85 \min 95 \% \mathrm{~B}$; $93 \mathrm{~min} 95 \% \mathrm{~B}$; $95 \min 10 \% \mathrm{~B} ; 100 \mathrm{~min} 10 \% \mathrm{~B}$, where $\mathrm{A}=0.1 \%$ formic acid in water and $\mathrm{B}=0.1 \%$ formic acid in $\mathrm{ACN}$. The effluent was directed into the ion spray source of a QSTAR XL hybrid mass spectrometer (Applied Biosystems) scanning from 300 to $1600 \mathrm{~m} / \mathrm{z}$. The top three most abundant, multiply-charged peptides were selected for MS/MS analysis $(80-1600 \mathrm{~m} / z)$. The resulting data were searched against the International Protein Index (IPI) rat database v 3.48 (European Bioinformatics Institute, Hinxton, Cambridge, UK) using ProteinPilot 2.0 (Applied Biosystems). Search parameters were: enzyme, trypsin; cys alkylation, iodoacetamide; search effort, rapid. Data summaries containing detailed information on all matched spectra are available for all six runs as Supporting Information material (Supporting Information Table 2). In cases where the protein names assigned by IPI were deemed too general we adopted the protein names used in linked pages in either the Rat Genome Database (RGD) or UniProt databases, when available. Alternatively, the amino acid sequence of the matched protein was used to perform a BLAST search (http://blast.ncbi.nlm.nih.gov), which typically resulted in identification of an identical protein with an established name. False-discovery rates for protein identifications were estimated by performing identical searches using the same database with all sequences reversed.

\subsection{Proteomic calculations (diabetic versus control comparisons)}

The ProteinPilot peptide summaries from six individual diabetic-versus-control comparisons were processed manually, according to our standard procedure with minor modifications $[9,10]$. Briefly, all spectra with a used score $=0$ were excluded, as were spectra matched to a peptide with a $\mathrm{COOH}$ terminal proline (known to interfere with the $116 \mathrm{~m} / z$ iTRAQ label, $[9,10])$. Remaining spectra were then normalized so that the sums of the areas were equal for each label within each LC-MS/MS run (uniform normalization). Alternatively, proteins of known mitochondrial localization were normalized separately from those of non-mitochondrial localization (mt/non-mt normalization). For each run, the sum of all peak areas for all spectra matched to a unique protein was then calculated for each label separately. These values were then log transformed and diabetic versus control log ratios obtained for all identified proteins in each of the six individual LC-MS/MS runs. Note that proteins identified with an unused score $<2$, the chosen cut-off, were excluded. The displayed, final diabetes-versus-control ratios represent the average of all log ratios from the individual experiments. Statistical significance of differences was tested for all proteins found in at least three LC-MS/MS runs (two-tailed one sample t-test, GraphPad Prism 5.02, San Diego, CA, USA); $p<0.05$ was considered significant and $p<0.10$ trending. SEM values were calculated for each protein from the log-transformed ratios and used to determine confidence intervals (average \pm SEM) for all quantified proteins in linear space. For cluster analysis, the complete list of 398 unique proteins was scanned manually for proteins that fitted into any of the 15 functional clusters of interest. Care was taken to construct clusters containing only proteins that would be expected to change in the same direction to any set of circumstances (see Supporting Information Table 3 for information regarding proteins within each cluster). The log ratios for all observations of all proteins within each cluster were then analyzed as a group and $p$-values calculated as described above. This enabled relative quantification of several mitochondrial electron transport system (ETS) protein complexes, for which quantification at the individual protein level could not be performed since the protein subunits were identified in less than three experiments.

\subsection{Western blotting}

Proteins obtained as described above were separated by SDS-PAGE on gradient (4-12\%) gels (50 $\mathrm{g}$ ger lane) and transferred to PVDF membranes. Western blotting was performed following standard protocols using primary antibodies raised against lamin-A, carbonic anhydrase (CA) 3, fibromodulin or S100 calcium-binding protein A10 (S100A10) (all from Santa Cruz Biotechnologies, Santa Cruz, CA, USA). Secondary peroxidase-linked anti-mouse or anti-rabbit IgG antibodies were used to generate immunocomplexes that were visualized using ECL Plus ${ }^{\text {TM }}$ (GE Healthcare, Waukesha, WI, USA). Images were captured using a LAS-3000 Luminescent Image Analyser (Fuji Photo Film, Tokyo, Japan) and band intensities were quantified by 
ImageJ (freely available at http://rsb.info.nih.gov/ij/). Statistical differences between groups were evaluated using two-tailed t-tests (GraphPad Prism 5.02).

\subsection{Enzyme assays}

Aortic tissue was homogenized in extraction buffer (50 mmol/L Tris, pH 7.4, $150 \mathrm{mmol} / \mathrm{L} \mathrm{NaCl}, 5 \mathrm{mmol} / \mathrm{L}$ EDTA, $10 \mathrm{mmol} / \mathrm{L}$ DTT and $1 \%$ Triton X-100) with addition of a protease inhibitor mixture (Roche, Indianapolis, IN, USA). The homogenate was then centrifuged at $110000 \times \mathrm{g}$ at $4{ }^{\circ} \mathrm{C}$ for $1 \mathrm{~h}$ and the supernatant was used for the assay. Total CA activity was assayed by the imidazole-Tris technique [11] with some modifications. Briefly, $300 \mu \mathrm{g}$ protein per sample was incubated in a sealed container with dry ice for $5 \mathrm{~min}$, and $0.5 \mathrm{~mL}$ of imidazole buffer $(20 \mathrm{mmol} / \mathrm{L}$ imidazole, $5 \mathrm{mmol} / \mathrm{L}$ Tris, $0.2 \mathrm{mmol} / \mathrm{L}$ p-nitrophenol, $\mathrm{pH}$ 9.5) was added to start the reaction. Time elapsed was recorded until the yellow reaction mixture became colorless. The CA activity was determined by the equation: CA (units/mg protein $)=\log (B / S) / P / \log 2$, where $B$ and $S$ are the times recorded for the paired, boiled control and test samples, respectively and $P$ is the protein concentration. Total antioxidant potential in extracts was assayed using the Bioxytech AOP- $490^{\text {TM }}$ kit (Oxis International, Foster City, CA, USA). Semicarbazide-sensitive amine oxidase (SSAO) activity was determined using the Fluoro SSAO ${ }^{\mathrm{TM}}$ detection kit (Bachem Americas, Torrance, CA, USA) with enzyme preparation carried out according to a published method [12]. The statistical significance of pairwise differences between group means was determined for all enzyme assays using twotailed $t$-tests (GraphPad Prism 5.02), and statistical significance was set at $p<0.05$.

\section{$3 \quad$ Results}

\subsection{Animal and tissue parameters}

Rats became diabetic within $48 \mathrm{~h}$ after STZ injection and hyperglycaemia persisted throughout the 16-wk experimental period. Blood glucose levels in diabetic rats were on average $31.8 \mathrm{mM}(p<0.0001$; Table 1$)$ and, at the end of wk 16 , bodyweight gain was fourfold less in diabetic rats than in control rats $(72.3 \pm 19.0 \mathrm{~g}$ versus $285.6 \pm 10.6 \mathrm{~g}, p<0.0001)$. Values for both the inner diameters and cross-sectional areas were significantly lower for diabetic aortas than corresponding control values (Table 1). Histological analysis showed a marked decrease in the wave-form present in elastic laminae (stained black with Movat's pentachrome) of diabetic aortic tissue (Fig. 1), consistent with decreased elasticity of the wall. There was no significant difference in total protein content between groups of diabetic and control aortas $(57.3 \pm 5.3$ and $51.4 \pm 2.6 \mathrm{mg} / \mathrm{g}$ wet weight, respectively; $p=$ n.s.).

\section{2 iTRAQ/MudPIT}

A total of 167532 spectra were generated across six independent pair-wise LC-MS/MS runs, and 39571 spectra were matched to 392 unique proteins (a complete list is available from the authors upon request). Of these, 208 proteins were identified with an unused score $\geq 2.0$ in at least three independent LC-MS/MS runs and with sufficient iTRAQ label to enable reliable relative quantitation between the diabetic and non-diabetic aortic tissue (Supporting Information Table 1). The false-discovery rate at this cut-off was

Table 1. Parameters of animals and aortas in control and diabetic rats

\begin{tabular}{lrc}
\hline Measurement & \multicolumn{1}{l}{ Control } & \multicolumn{1}{c}{ Diabetic } \\
\hline Blood glucose $(\mathrm{mM})$ & $4.8 \pm 0.2(9)$ & $31.8 \pm 0.3^{* *}(7)$ \\
Body weight $(\mathrm{g})$ & $515.6 \pm 10.6(9)$ & $302.3 \pm 19.0^{*}(7)$ \\
Inner diameter $(\mathrm{mm})$ & $1.8 \pm 0.1(6)$ & $1.6 \pm 0.1^{*}(6)$ \\
Cross section area $\left(\mathrm{mm}^{2}\right)$ & $2.9 \pm 0.2(5)$ & $2.6 \pm 0.1^{*}(5)$ \\
Medial thickness $(\mu \mathrm{m})$ & $106.3 \pm 4.8(6)$ & $98.8 \pm 4.6(6)$ \\
Perimeter $(\mathrm{mm})$ & $6.2 \pm 0.2(6)$ & $6.3 \pm 0.1(6)$ \\
\hline
\end{tabular}

${ }^{*} p<0.05,{ }^{* *} p<0.01$ versus non-diabetic rats, respectively. Individual replicates $(n)$ are shown in parentheses.

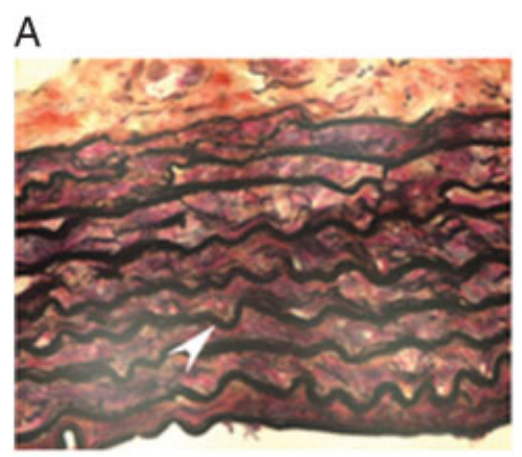

B

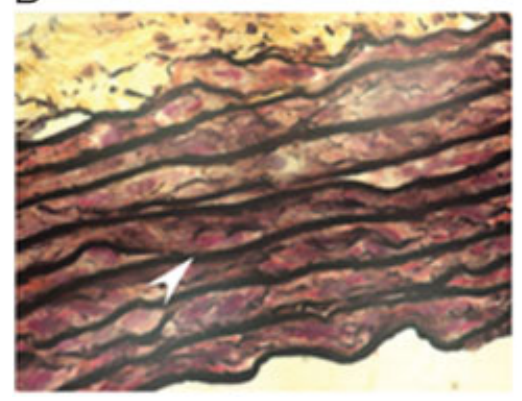

Figure 1. Representative cross-sections of descending aortas from non-diabetic control (A) and diabetic (B) rats stained with Movat's Pentachrome ( $n=6 / g r o u p)$. Arrows mark elastic laminae (stained black). Note straightening of the wave-like appearance in (B) compared with (A). Each micrograph is oriented with the lumen below and the adventitia above; original magnification, $\times 40$. 
$1.4 \pm 0.3 \%$ with only one reversed hit found in three or more runs.

Proteins with iTRAQ ratios showing significant $(p<0.05)$ or trending towards $(p<0.10)$ changes in diabetic aortic tissue are listed and assigned to functional groups in Table 2. A total of 64 identified proteins (22 of those found in at least three runs) were identified as being primarily or exclusively mitochondrial in origin. Although the relative abundance was significantly different for only one mitochondrial protein itself and there were trends towards decrease in two additional proteins, mitochondrial proteins as a group showed disproportionately low diabetic-versus-control ratios. Specifically, average and median diabetic versus control ratios were 0.78 and 0.79 for the group of all 64 mitochondrial proteins while corresponding figures for the 328 non-mitochondrial proteins were 0.97 and 1.00. The difference (mitochondrial versus non-mitochondrial average ratios) was statistically significant $(p<0.0001)$, indicating that the total amount of proteins of mitochondrial origin was lower in diabetic aorta as compared with non-diabetic controls. This prompted the use of an additional, complementary, normalization approach better suited to explore changes in abundance within the mitochondrial subset of proteins (see Section 2). Although this alternative approach made minimal difference to the protein ratios in the nonmitochondrial group, mitochondrial heat shock protein 60 and its companion chaperone 10 trended towards decrease $(p \leq 0.10$; Table 2) with diabetes, and mitochondrial ES1 protein homologue decreased significantly in diabetic aorta (78\% of controls, $p<0.05$ ).

As a complement to the assessment of changes in abundance of each of the 208 individual proteins, we also performed a functional cluster analysis that included those proteins found only once or twice (Table 3). All seven mitochondria-based clusters were lower in diabetes compared with controls when a uniform scaling was applied. Normalizing mitochondrial proteins to total mitochondrial material prior to obtaining ratios rendered the diabetes versus control comparison for several mitochondrial clusters non-significant and in all cases closer to a 1:1 ratio. Of 15 clusters analyzed, five were found to be significantly different in diabetic aortas, and a further three exhibited $p \leq 0.10$. Notably, ETS complexes III and IV were significantly lower in diabetic aortas even after scaling to total mitochondrial protein, while complex V (ATP synthase) was proportional to the level of mitochondrial material in either group. Proteins involved in mitochondrial reactive oxygen species (ROS) defence were unchanged as a group while non-mitochondrial intracellular ROS-related proteins trended towards elevation in diabetic aorta $(p \leq 0.10)$. Furthermore, both myosin and actin clusters were unaltered, although it is clear that certain individual myosin isoforms were increased in diabetes.

\subsection{Western blot and enzyme assays}

To validate our proteomic results, we measured protein levels of four selected targets of interest in aortic extracts by
Western blotting. Consistent with our proteomic results, Western analysis confirmed increased protein levels of CA3, lamin-A and S100A10, and decreased levels of fibromodulin (Fig. 2) in diabetic aorta as compared with control tissue. Furthermore, both total CA and SSAO activities were significantly increased in diabetic aortic tissue (Fig. 3). In addition, total antioxidant potential was on average a third lower $(p=0.065)$ in diabetic aortas than in corresponding control tissues (Fig. 3).

\section{Discussion}

STZ-induced diabetes in rats is a reliable model for the study of processes leading to the diabetic complications, including cardiomyopathy, nephropathy and arteriopathy. Rats treated with a similar dose of STZ as employed here were previously reported to develop arterial lesions after 4 wk diabetes [13]. Here, STZ-treated rats had the expected elevation of blood glucose and decreased bodyweight and also demonstrated significant flattening of the normally wave-shaped aortic elastic laminae, consistent with stiffening and loss of elasticity. Thus, our model replicated this important aspect of diabetic arteriosclerosis.

The aorta is a large elastic artery and as such has many properties in common with smaller arteries and arterioles. Hence, the aortic proteome may provide insights relevant to the development of diabetes-associated pathology in these other, smaller vessels, which are less accessible for proteomic study owing to mass limitations. Rats with severe diabetes for the duration studied here develop arteriosclerosis in large arteries such as the aorta, but not the focal lesions of atherosclerosis. Thus, the current findings are considered primarily relevant to arteriosclerosis. While proteomic techniques have previously been used to study cardiac diseases, the proteome of the vascular system remains poorly explored [14], with only one available report for diabetic aortic tissue that employed a preliminary protein-chip based study that did not identify or quantify specific proteins [15]. To our knowledge, the present study is the first comprehensive proteomic study of the diabetic aorta, and the first to employ iTRAQ/MudPIT technology for proteomic analysis of aortic proteins in diabetic arteriosclerosis.

Specifically, we identified significant, or trends towards, diabetes-evoked alterations in 51 of 208 unique aortic proteins, many of which have not previously been reported to be associated with diabetes or the development of diabetic arteriosclerosis.

Atherosclerosis is a focal diffuse inflammatory condition characterized by increased oxidative stress, which leads to localized arterial wall thickening and eventually to stenosis through the build-up of plaques containing fats, cholesterol and calcium deposits inside the affected arteries (see [16] for a recent review). In contrast, atherosclerotic plaques are not present in 'pure' arteriosclerosis, which is characterized by 


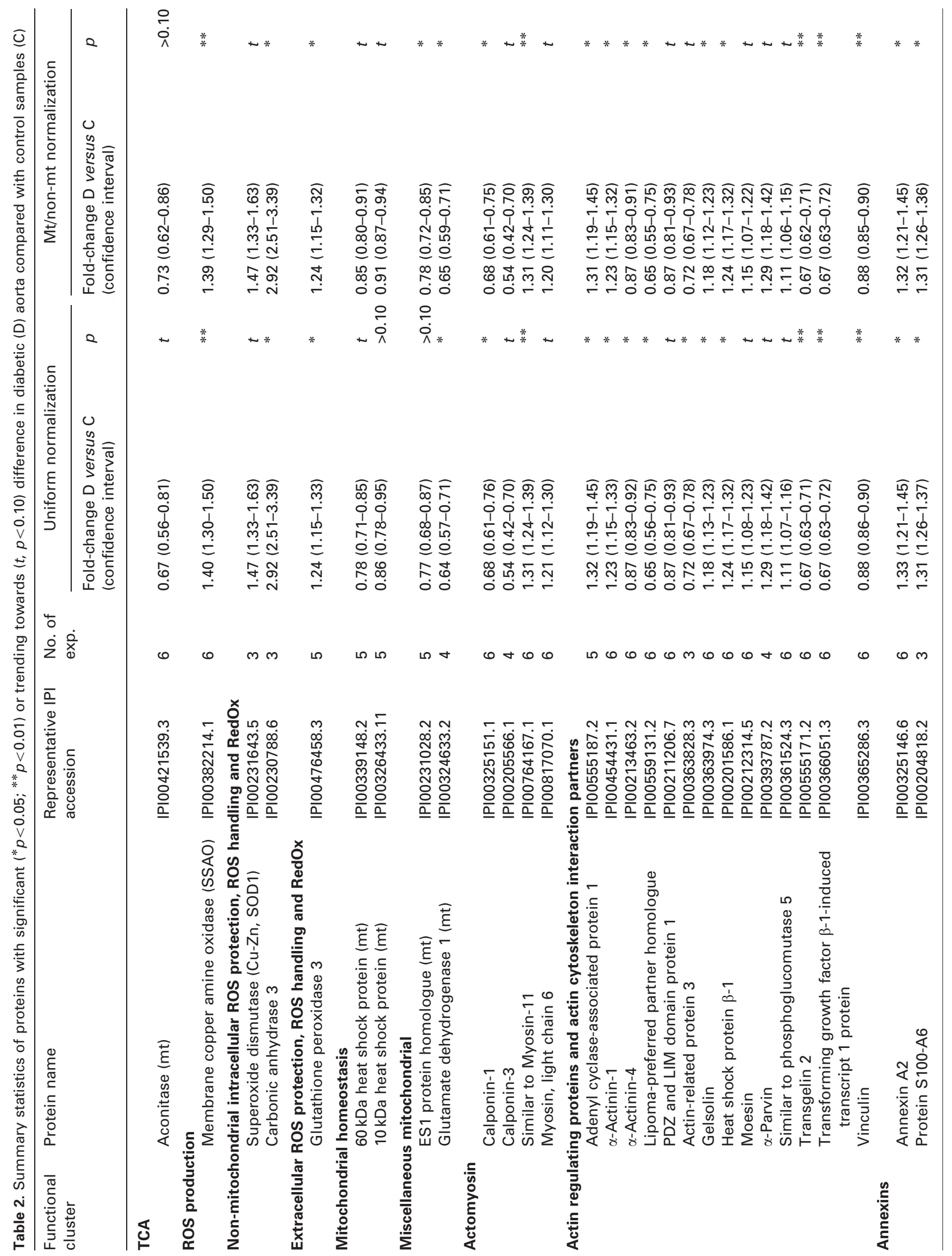




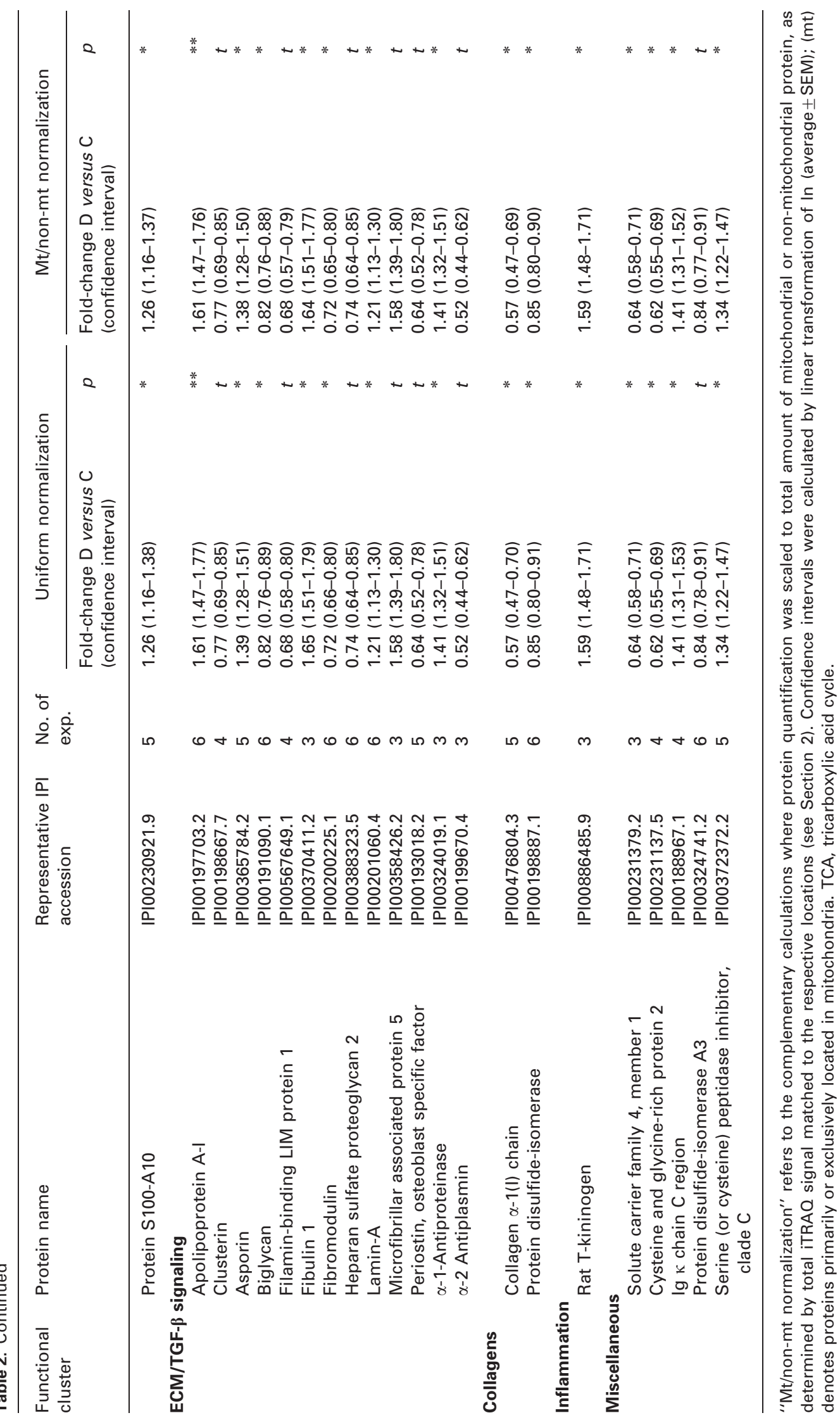


Table 3. Cluster analysis comparing relative abundance in diabetic (D) versus control (C) aortic tissue of 94 proteins allocated to 15 functional clusters

\begin{tabular}{|c|c|c|c|c|c|}
\hline \multirow[t]{2}{*}{ Functional cluster } & \multirow{2}{*}{$\begin{array}{l}\text { Total no. of observations } \\
\text { (no. of proteins in cluster) }\end{array}$} & \multicolumn{2}{|c|}{ Uniform normalization } & \multicolumn{2}{|c|}{ Mt/non-mt normalization } \\
\hline & & $\begin{array}{l}\text { Fold-change D:C } \\
\text { (confidence interval) }\end{array}$ & $p$ & $\begin{array}{l}\text { Fold-change D:C } \\
\text { (confidence interval) }\end{array}$ & $p$ \\
\hline Glycolysis and fermentation & $55(11)$ & $0.89(0.85-0.94)$ & $*$ & $0.89(0.85-0.94)$ & $*$ \\
\hline Complex III (mt) & $6(3)$ & $0.66(0.60-0.73)$ & $*$ & $0.71(0.65-0.79)$ & $*$ \\
\hline Complex IV (mt) & $9(5)$ & $0.63(0.55-0.73)$ & $*$ & $0.69(0.60-0.78)$ & * \\
\hline Complex V (mt) & $26(9)$ & $0.90(0.85-0.94)$ & $*$ & $0.99(0.96-1.02)$ & $>0.10$ \\
\hline Mitochondrial homeostasis (mt) & $13(5)$ & $0.87(0.82-0.93)$ & $t$ & $0.95(0.90-1.00)$ & $>0.10$ \\
\hline TCA (mt) & $21(5)$ & $0.80(0.74-0.86)$ & $*$ & $0.85(0.80-0.91)$ & * \\
\hline $\mathrm{FAO}(\mathrm{mt})$ & $15(7)$ & $0.73(0.64-0.83)$ & $*$ & $0.80(0.70-0.91)$ & $>0.10$ \\
\hline $\begin{array}{l}\text { Mitochondrial ROS protection, ROS } \\
\text { handling and RedOx (mt) }\end{array}$ & $10(4)$ & $0.91(0.86-0.97)$ & $>0.10$ & $0.97(0.89-1.06)$ & $>0.10$ \\
\hline $\begin{array}{l}\text { Non-mitochondrial intracellular ROS } \\
\text { protection, ROS handling and RedOx }\end{array}$ & $21(6)$ & $1.20(1.08-1.32)$ & $t$ & $1.19(1.08-1.32)$ & $t$ \\
\hline Intermediate filaments & $12(2)$ & $1.22(1.06-1.39)$ & $>0.10$ & $1.21(1.06-1.39)$ & $>0.10$ \\
\hline Myosin & $48(11)$ & $0.96(0.87-1.06)$ & $>0.10$ & $0.96(0.87-1.06)$ & $>0.10$ \\
\hline Actin & $14(3)$ & $0.93(0.83-1.04)$ & $>0.10$ & $0.93(0.83-1.04)$ & $>0.10$ \\
\hline Collagens & $38(8)$ & $0.85(0.78-0.93)$ & $t$ & $0.85(0.78-0.93)$ & $t$ \\
\hline Tubulin & $20(4)$ & $1.04(0.97-1.12)$ & $>0.10$ & $1.04(0.97-1.11)$ & $>0.10$ \\
\hline Annexins & $41(8)$ & $1.15(1.09-1.21)$ & $*$ & $1.14(1.09-1.21)$ & $*$ \\
\hline
\end{tabular}

"Mt/non-mt normalization" refers to the complementary calculations where protein quantification was scaled to total amount of mitochondrial or non-mitochondrial protein, as determined by total iTRAQ signal matched to the respective locations (see Section 2). (mt) denotes clusters of proteins with exclusively mitochondrial localization. ${ }^{*}<0.05, t=<0.10$. TCA, tricarboxylic acid cycle; FAO, fatty acid oxidation.

A
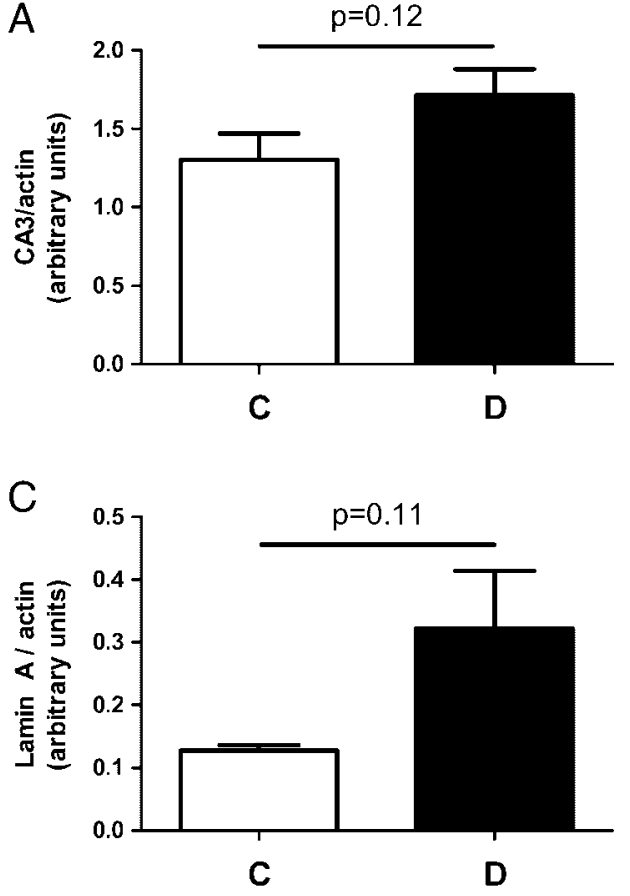

B
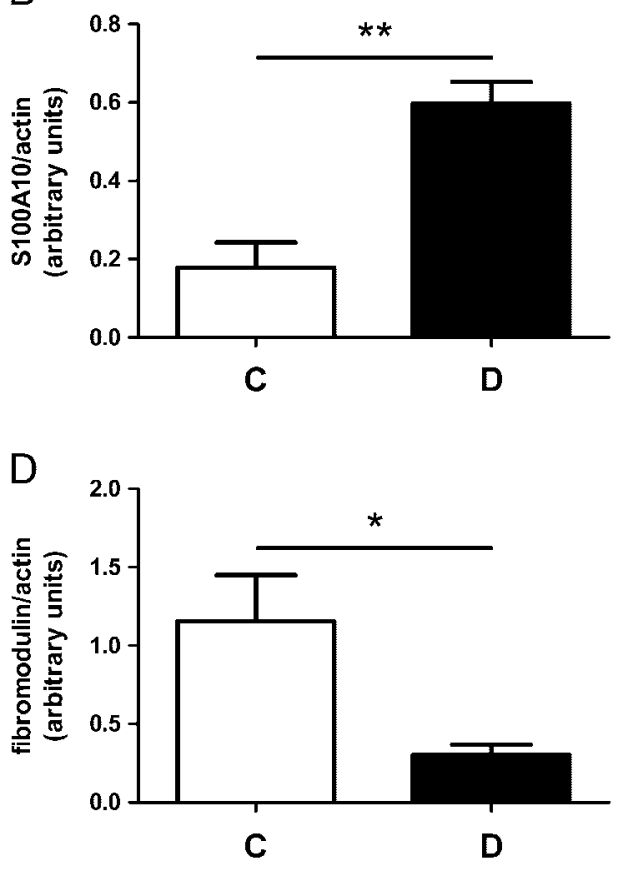

Figure 2. Densitometry of Western blots for selected proteins normalized to $\beta$-actin in aortic homogenates from non-diabetic control (C) and diabetic (D) rats. $n=5$ per group for each protein tested; ${ }^{*} p<0.05 ;{ }^{* *} p<0.01$. the diffuse hardening, stiffness and thickening of the arterial wall as was the case here in STZ-diabetic rats.

Atherosclerosis does, however, occur frequently in diabetic patients, where it has been reported to result in specific proteomic changes. The alterations in CA3 [17], lamin-A [18] and apolipoprotein A-1 identified in the present study are consistent with prior studies of atherosclerosis reported elsewhere [19-21]. Our finding of significantly 
A

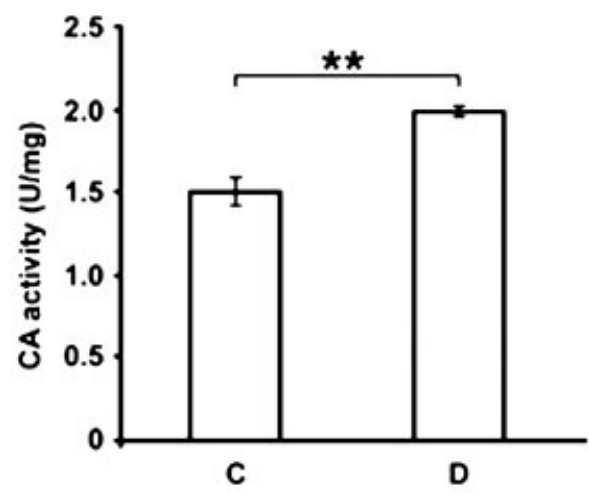

B
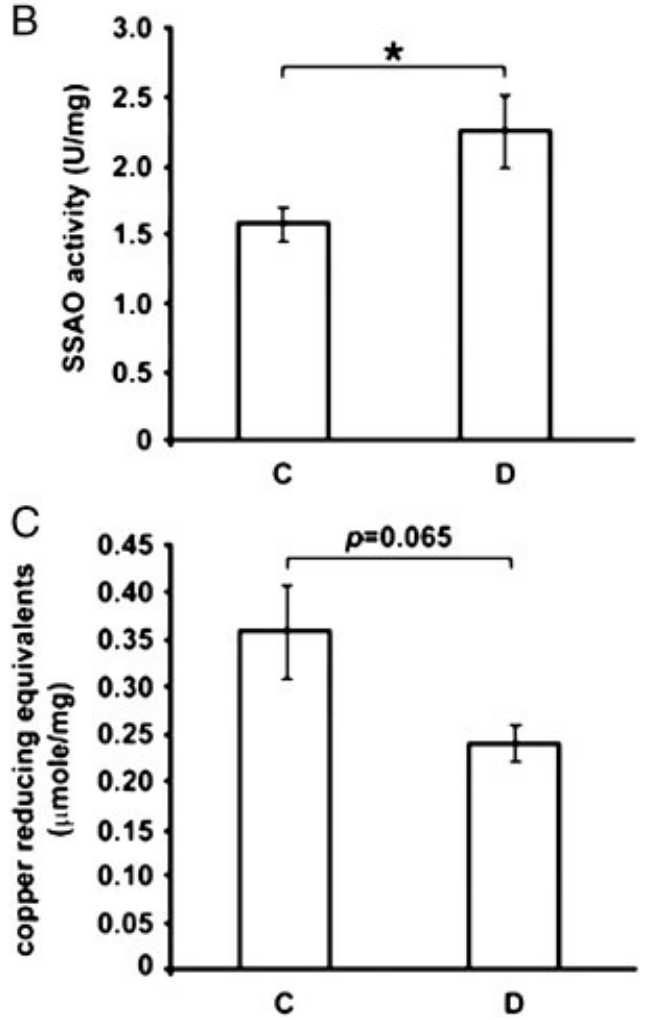

Figure 3. Enzymatic activities of (A), total CA and (B), SSAO; and (C) total antioxidant potential in aortic tissue from diabetic rats and non-diabetic controls. $n=5-7$ per group; ${ }^{*} p<0.05$; $*^{* *} p<0.01$.

elevated levels of apolipoprotein A-1 contrasts with the more than threefold reduction observed in urine from patients with type 2 diabetes [22], suggesting that this protein is retained to a greater extent in the diabetic state. Also consistent with reports of atherosclerotic damage, we found elevated levels of several proteins implicated in inflammation, including the inflammatory marker T-kininogen (1.6fold higher in diabetic aorta, $p<0.05$ ) and anti-inflammatory annexin A2 and its ligand, protein S100A10 (both 1.3-fold higher in diabetic aorta, $p<0.05)$. These studies point to similarities in the processes that lead to or cause arterio- sclerosis and atherosclerosis in the arteries of diabetic individuals.

Another significant finding here was that aortic tissue from diabetic animals contained markedly lower amounts of mitochondrial proteins, when considered in aggregate, than did aortic tissue from non-diabetic control animals. Furthermore, each mitochondrion appeared to contain on average significantly lower amounts of several subunits from each of ETS complexes III and IV, whereas levels of intra-mitochondrial subunits of ATP synthase (complex V) and proteins implicated in mitochondrial homeostasis and protection against ROS-mediated damage were unaltered. These findings appear consistent with impaired flow of electrons through the ETS, which is generally thought to promote ROS release from ETS complexes I and III [23]. Indeed, a strong correlation between oxidative stress, diabetes and vascular diseases has been reported [24, 25], and there is some evidence to suggest increased mitochondrial ROS production in aortic endothelial cells exposed to high glucose [26]. If ROS production is increased above the capacity of cellular defence systems in diabetic aorta, a concomitant increase in ROS scavenging or processing proteins might also be expected. However, mitochondrial aldehyde dehydrogenase, a key buffer of mitochondrial ROS damage, and a corresponding functional cluster of four mitochondrial ROS-protective proteins, remained largely unaltered in diabetic aortas here.

Elevated ROS levels may also originate from extramitochondrial sources. We found 1.4-fold higher $(p<0.05)$ levels of the membrane copper amine oxidase, SSAO, in diabetic aorta by both iTRAQ/MudPIT and enzymatic assay. This $\mathrm{H}_{2} \mathrm{O}_{2}$-forming enzyme is well-known to be elevated in diabetes and may directly contribute to vascular complications in diabetes [27-29].

Certain ROS-responsive enzymes were also elevated in diabetic aorta. Extracellular glutathione peroxidase 3 was modestly increased $(1.2$-fold, $p<0.05)$, while the carbonic anhydrase isoform CA3 was substantially elevated (2.9-fold, $p<0.05)$. CA3 differs from other carbonic anhydrase isoforms (CA1, 2 and 4), in that its turnover rate is lower and it can act as an antioxidant through glutathionylation [30]. The marginal increase in total CA activity (1.3-fold) in diabetic aorta relative to CA3 levels, and the lower total antioxidant potential, is consistent with a response to elevated ROS in diabetic aortic extracts.

Arteriosclerosis reportedly involves fibrosis [2], a process thought to depend largely on TGF- $\beta$ signaling [31, 32]. TGF$\beta$ protein and activity are elevated in some diabetic tissues, and experimental diabetes is well known to lead to vascular hypertrophy [33]. In diabetic vascular fibrosis, elevated levels of ECM components, e.g. collagen, are typically apparent [34-36]. Here, ECM proteins such as fibronectin, decorin and at least four types of collagen showed no change while collagen $\alpha$ (I) was depressed (57\% of control aorta, $p<0.05$ ). Notably, multiple targets of TGF- $\beta$, e.g. TGF- $\beta$ - 1 -induced transcript 1 protein, calponin-1 [37], transgelin 2 [38], 
periostin [39], fibromodulin [40], and cysteine and glycinerich protein 2 [41] were all present at significantly lower levels in diabetic aorta. These findings strongly suggest that TGF- $\beta$ signaling is decreased rather than increased in STZdiabetic aortas, which contrasts with reports of elevated TGF- $\beta$ signaling in the STZ diabetic model in other tissues, for example the myocardium. We note that the TGF- $\beta$ inhibitory factors asporin and gelsolin were elevated (1.4and 1.2-fold respectively, both $p<0.05)$, and that these proteins could potentially modulate the local TGF- $\beta$ response in aortic tissue $[42,43]$.

Atherosclerosis has previously been linked to changes in cytoskeletal proteins in different cell types [44, 45]. Although we found no substantial changes in abundance of cytoplasmic actin ( $\beta$ or $\gamma$ isoforms), several proteins involved in dynamic regulation of actin filaments were significantly altered. Actin organizing heat shock protein $\beta-1$ was elevated 1.2 -fold $(p<0.05)$, a finding previously correlated with protection from atherosclerosis [46]. Furthermore, we detected elevated levels of adenylyl cyclase-associated protein 1 (1.3-fold higher, $p<0.05)$ and gelsolin, (1.2-fold higher, $p<0.05)$, consistent with increased demands for more rapid actin turnover and elevated cell mobility required for wound healing $[47,48]$. Notably gelsolin can also be upregulated in response to oxidative stress [49]. Lower levels of transgelin 2 may also serve to enhance actin depolymerization [38], and levels of the bundling protein $\alpha$-actinin- 1 were elevated while the $\alpha$-actinin- 4 isoform was decreased, as were actinininteracting lipoma-preferred partner, and PDZ and LIM domain protein 1 .

$\alpha$-Actin and myosin constitute the actomyosin myofibrils, which mediate arterial wall contraction. Here, we detected 1.3-fold increased levels of smooth muscle heavy chain myosin-11 in diabetic aortic tissues $(p<0.05)$, and 1.2-fold higher levels of myosin light chain $6(p<0.10)$. We also found depressed levels of calponin-1 $(p<0.05)$ and $-3 \quad(p<0.10)$ levels. Calponins interact with actin to lower actomyosin MgATPase activity [50], and lower calponin levels can be consistent with an aortic response in diabetic animals attempting to increase contractile capacity. Myofibrillar actin has also been directly linked to $\mathrm{Ca}^{2+}$ storage and signaling [51], and the changes we observed may reflect responses to calcification and increased aortic stiffening in diabetes.

Proteomic studies on vascular tissue and diabetes-related changes are practically non-existent; so the identification here of processes modified by induced diabetes is novel, highly relevant and an appropriate prerequisite for studies on human tissue and smaller blood vessels. The current study was designed to demonstrate proof-of-principle, and thus to pave the way towards the development of proteomic methods for application in the investigation of the mechanisms of common arterial diseases in patients. Many of the common/widespread chronic diseases of adulthood, including diabetes mellitus and its cardiovascular, renal and retinal complications, coronary artery disease/atheromatosis, stroke, essential hypertension, Alzheimer's disease and vascular dementia, are all basically manifestations of underlying arterial diseases [52]. In all of these common diseases, precise characterizations of the component pathogenic pathways/mechanisms remain to be developed. The current study is seen as paving the way towards improved characterization of these diseases particularly their underpinning arterial molecular pathobiology - as a necessary step in defining new targets for the development of novel experimental pharmacotherapies. As an exemplar, our group has been using proteomics-based strategies to investigate the role of dysregulated copper metabolism in the mechanism of diabetic organ damage (cardiomyopathy, nephropathy, arteriopathy and retinopathy). These investigations have led to the invention of a new method for rebalancing tissue metal distribution in diabetes, which in turn leads/causes organs to regenerate $[53,54]$. The application of proteomics-based approaches to the elucidation of human disease mechanisms is thus demonstrably capable of generating new experimental therapies for the treatment of arterial diseases, as exemplified by those in diabetes.

In summary, here we have identified many proteins and pathways newly implicated in the pathogenesis of diabetic arteriosclerosis. Key findings include decreased mitochondrial proteins with disproportional deficiencies in subunits of ETS complexes III and IV, increased oxidative stress-response proteins (probably responding to stress from a non-mitochondrial source) and multiple changes in levels of actin cytoskeleton remodeling proteins and actomyosin. The lack of changes consistent with fibrosis and pro-fibrotic TGF- $\beta$ signaling suggests that the arteriosclerosis in this investigation was at a pre-fibrotic stage. This proteomic characterization represents a major step towards understanding the molecular circumstances that mediate the development of diabetic arteriosclerosis in the STZ-diabetic model. This study could also facilitate the identification of pharmacological targets or biomarkers suitable for targeting and/or monitoring therapeutic responses of arteriosclerosis in its early phases following disease onset.

The authors thank C. A. Tse and V. Tintinger (School of Biological Sciences, University of Auckland, Auckland, New Zealand) for administrative assistance and manuscript editing, and technical assistance, respectively. This work was funded by grants from the Endocore Research Trust; the Maurice and Phyllis Paykel Trust; Lottery Health (New Zealand); The Auckland Medical Research Foundation; the University of Auckland; the Department of Education (New Zealand) through a grant to the Maurice Wilkins Centre of Excellence for Molecular Biodiscovery; by program grants to G. J. C. from the Foundation for Research Science and Technology, New Zealand and from the Health Research Council of New Zealand.

The authors have declared no conflict of interest. 


\section{References}

[1] Nathan, D. M., Lachin, J., Cleary, P., Orchard, T. et al., Intensive diabetes therapy and carotid intima-media thickness in type 1 diabetes mellitus. N. Engl. J. Med. 2003, 348, 2294-2303.

[2] Selvin, E., Najjar, S. S., Cornish, T. C., Halushka, M. K., A comprehensive histopathological evaluation of vascular medial fibrosis: insights into the pathophysiology of arterial stiffening. Atherosclerosis 2010, 208, 69-74.

[3] Cruickshank, K., Riste, L., Anderson, S. G., Wright, J. S. et al., Aortic pulse-wave velocity and its relationship to mortality in diabetes and glucose intolerance: an integrated index of vascular function? Circulation 2002, 106, 2085-2090.

[4] Nakashima, Y., Fujii, H., Sumiyoshi, S., Wight, T. N., Sueishi, K., Early human atherosclerosis: accumulation of lipid and proteoglycans in intimal thickenings followed by macrophage infiltration. Arterioscler. Thromb. Vasc. Biol. 2007, 27, 1159-1165.

[5] Mahfouz Badran, H., Elnoamany, M., Impact of type 2 diabetes mellitus on aortic elastic properties in normotensive diabetes: Doppler tissue imaging study. J. Am. Soc. Echocardiogr. 2006, 19, 1471-1481.

[6] Fukuda, G., Khan, Z. A., Barbin, Y. P., Farhangkhoee, H. et al., Endothelin-mediated remodeling in aortas of diabetic rats. Diabetes Metab. Res. Rev. 2005, 21, 367-375.

[7] Gong, D., Lu, J., Chen, X., Choong, S. Y. et al., Molecular changes evoked by triethylenetetramine treatment in the extracellular matrix of the heart and aorta in diabetic rats. Mol. Pharmacol. 2006, 70, 2045-2051.

[8] Movat, H. Z., Demonstration of all connective tissue elements in a single section. AMA Arch. Pathol. 1955, 55, 289-295.

[9] Jüllig, M., Hickey, A. J., Middleditch, M. J., Crossman, D. J. et al., Characterization of proteomic changes in cardiac mitochondria in streptozotocin-diabetic rats using iTRAQ ${ }^{\mathrm{TM}}$ isobaric tags. Proteomics Clin. Appl. 2007, 1, 565-576.

[10] Jüllig, M., Hickey, A. J., Chai, C. C., Skea, G. L. et al., Is the failing heart out of fuel or a worn engine running rich? A study of mitochondria in old spontaneously hypertensive rats. Proteomics 2008, 8, 2556-2572.

[11] Brion, L. P., Schwartz, J. H., Zavilowitz, B. J., Schwartz, G. J., Micro-method for the measurement of carbonic anhydrase activity in cellular homogenates. Anal. Biochem. 1988, 175, 289-297.

[12] Ochiai, Y., Itoh, K., Sakurai, E., Tanaka, Y., Molecular cloning and characterization of rat semicarbazidesensitive amine oxidase. Biol. Pharm. Bull. 2005, 28, 413-418.

[13] Lu, Y. L., Hu, S. J., Shen, Z. J., Shao, Y. C., Changes of macrovascular endothelial ultrastructure and gene expression of endothelial nitric oxide synthase in diabetic rats. Chin. Med. J. (Engl.) 2004, 117, 1165-1169.

[14] Mayr, M., Mayr, U., Chung, Y. L., Yin, X. et al., Vascular proteomics: linking proteomic and metabolomic changes. Proteomics 2004, 4, 3751-3761.
[15] Cho, W. C., Yip, T. T., Chung, W. S., Leung, A. W. et al., Differential expression of proteins in kidney, eye, aorta, and serum of diabetic and non-diabetic rats. J. Cell. Biochem. 2006, 99, 256-268.

[16] Victor, V. M., Rocha, M., Sola, E., Banuls, C. et al., Oxidative stress, endothelial dysfunction and atherosclerosis. Curr. Pharm. Des. 2009, 15, 2988-3002.

[17] Gamble, W., Atherosclerosis: the carbonic anhydrase, carbon dioxide, calcium concerted theory. J. Theor. Biol. 2006, 239, 16-21.

[18] Almofti, M. R., Huang, Z., Yang, P., Rui, Y., Proteomic analysis of rat aorta during atherosclerosis induced by high cholesterol diet and injection of vitamin D3. Clin. Exp. Pharmacol. Physiol. 2006, 33, 305-309.

[19] Panayiotou, A., Griffin, M., Georgiou, N., Bond, D. et al., ApoB/ApoA1 ratio and subclinical atherosclerosis. Int. Angiol. 2008, 27, 74-80.

[20] Wakabayashi, I., Masuda, H., Lipoprotein (a) as a determinant of arterial stiffness in elderly patients with type 2 diabetes mellitus. Clin. Chim. Acta 2006, 373, 127-131.

[21] Gunczler, P., Lanes, R., Soros, A., Verdu, L. et al., Coronary artery calcification, serum lipids, lipoproteins, and peripheral inflammatory markers in adolescents and young adults with type 1 diabetes. J. Pediatr. 2006, 149, 320-323.

[22] Rao, P. V., Lu, X., Standley, M., Pattee, P. et al., Proteomic identification of urinary biomarkers of diabetic nephropathy. Diabetes Care 2007, 30, 629-637.

[23] Murphy, M. P., How mitochondria produce reactive oxygen species. Biochem. J. 2009, 417, 1-13.

[24] Giugliano, D., Ceriello, A., Paolisso, G., Oxidative stress and diabetic vascular complications. Diabetes Care 1996, 19, 257-267.

[25] Liao, M., Liu, Z., Bao, J., Zhao, Z. et al., A proteomic study of the aortic media in human thoracic aortic dissection: implication for oxidative stress. J. Thorac. Cardiovasc. Surg. 2008, 136, 65-72, 72 e61-63.

[26] Sethi, A. S., Lees, D. M., Douthwaite, J. A., Dawnay, A. B., Corder, R., Homocysteine-induced endothelin-1 release is dependent on hyperglycaemia and reactive oxygen species production in bovine aortic endothelial cells. J. Vasc. Res. 2006, 43, 175-183.

[27] Boomsma, F., van den Meiracker, A. H., Winkel, S., Aanstoot, H. J. et al., Circulating semicarbazide-sensitive amine oxidase is raised both in type I (insulin-dependent), in type II (non-insulin-dependent) diabetes mellitus and even in childhood type I diabetes at first clinical diagnosis. Diabetologia 1999, 42, 233-237.

[28] Garpenstrand, H., Ekblom, J., Backlund, L. B., Oreland, L., Rosenqvist, U., Elevated plasma semicarbazide-sensitive amine oxidase (SSAO) activity in Type 2 diabetes mellitus complicated by retinopathy. Diabet. Med. 1999, 16, 514-521.

[29] Gokturk, C., Nilsson, J., Nordquist, J., Kristensson, M. et al., Overexpression of semicarbazide-sensitive amine oxidase in smooth muscle cells leads to an abnormal structure of the aortic elastic laminas. Am. J. Pathol. 2003, 163, 1921-1928. 
[30] Raisanen, S. R., Lehenkari, P., Tasanen, M., Rahkila, P. et al., Carbonic anhydrase III protects cells from hydrogen peroxide-induced apoptosis. FASEB J. 1999, 13, 513-522.

[31] Branton, M. H., Kopp, J. B., TGF-beta and fibrosis. Microbes Infect. 1999, 1, 1349-1365.

[32] Pohlers, D., Brenmoehl, J., Loffler, I., Muller, C. K. et al., TGF-beta and fibrosis in different organs - molecular pathway imprints. Biochim. Biophys. Acta 2009, 1792, 746-756.

[33] Gilbert, R. E., Rumble, J. R., Cao, Z., Cox, A. J. et al., Endothelin receptor antagonism ameliorates mast cell infiltration, vascular hypertrophy, and epidermal growth factor expression in experimental diabetes. Circ. Res. 2000, $86,158-165$.

[34] Rumble, J. R., Cooper, M. E., Soulis, T., Cox, A. et al., Vascular hypertrophy in experimental diabetes. Role of advanced glycation end products. J. Clin. Invest. 1997, 99, 1016-1027.

[35] Candido, R., Jandeleit-Dahm, K. A., Cao, Z., Nesteroff, S. P. et al., Prevention of accelerated atherosclerosis by angiotensin-converting enzyme inhibition in diabetic apolipoprotein E-deficient mice. Circulation 2002, 106, 246-253.

[36] Sun, H., Zhong, M., Miao, Y., Ma, X. et al., Impaired elastic properties of the aorta in fat-fed, streptozotocin-treated rats. Vascular remodeling in diabetic arteries. Cardiology 2009, 114, 107-113.

[37] Wicks, J., Haitchi, H. M., Holgate, S. T., Davies, D. E., Powell, R. M., Enhanced upregulation of smooth muscle related transcripts by TGF beta2 in asthmatic (myo) fibroblasts. Thorax 2006, 61, 313-319.

[38] Adam, P. J., Regan, C. P., Hautmann, M. B., Owens, G. K., Positive- and negative-acting Kruppel-like transcription factors bind a transforming growth factor beta control element required for expression of the smooth muscle cell differentiation marker SM22alpha in vivo. J. Biol. Chem. 2000, 275, 37798-37806.

[39] Horiuchi, K., Amizuka, N., Takeshita, S., Takamatsu, H. et al., Identification and characterization of a novel protein, periostin, with restricted expression to periosteum and periodontal ligament and increased expression by transforming growth factor beta. J. Bone Miner. Res. 1999, 14, 1239-1249.

[40] Levens, E., Luo, X., Ding, L., Williams, R. S., Chegini, N., Fibromodulin is expressed in leiomyoma and myometrium and regulated by gonadotropin-releasing hormone analogue therapy and TGF-beta through Smad and MAPK-mediated signalling. Mol. Hum. Reprod. 2005, 11, 489-494.

[41] Lin, D. W., Chang, I. C., Tseng, A., Wu, M. L. et al., Transforming growth factor beta up-regulates cysteine-rich protein 2 in vascular smooth muscle cells via activating transcription factor 2. J. Biol. Chem. 2008, 283, 15003-15014.
[42] Wang, D., Park, J. S., Chu, J. S., Krakowski, A. et al., Proteomic profiling of bone marrow mesenchymal stem cells upon transforming growth factor beta1 stimulation. $J$. Biol. Chem. 2004, 279, 43725-43734.

[43] Ikegawa, S., Expression, regulation and function of asporin, a susceptibility gene in common bone and joint diseases. Curr. Med. Chem. 2008, 15, 724-728.

[44] Desmouliere, A., Gabbiani, G., The cytoskeleton of arterial smooth muscle cells during human and experimental atheromatosis. Kidney Int. Suppl. 1992, 37, S87-S89.

[45] Holland, J. A., Goss, R. A., O'Donnell, R. W., Chang, M. M. et al., Low-density lipoprotein induced actin cytoskeleton reorganization in endothelial cells: mechanisms of action. Endothelium 2001, 8, 117-135.

[46] Rayner, K., Chen, Y. X., McNulty, M., Simard, T. et al., Extracellular release of the atheroprotective heat shock protein 27 is mediated by estrogen and competitively inhibits acLDL binding to scavenger receptor-A. Circ. Res. 2008, 103, 133-141.

[47] McGrath, J. L., Osborn, E. A., Tardy, Y. S., Dewey,C. F., Jr., Hartwig, J. H., Regulation of the actin cycle in vivo by actin filament severing. Proc. Natl. Acad. Sci. USA 2000, 97, 6532-6537.

[48] Moriyama, K., Yahara, I., Human CAP1 is a key factor in the recycling of cofilin and actin for rapid actin turnover. J. Cell Sci. 2002, 115, 1591-1601.

[49] Ji, L., Chauhan, A., Chauhan, V., Upregulation of cytoplasmic gelsolin, an amyloid-beta-binding protein, under oxidative stress conditions: involvement of protein kinase C. J. Alzheimers Dis. 2009, 19, 829-838.

[50] Winder, S. J., Walsh, M. P., Smooth muscle calponin. Inhibition of actomyosin MgATPase and regulation by phosphorylation. J. Biol. Chem. 1990, 265, 10148-10155.

[51] Lange, K., Gartzke, J., A critical comparison of the current view of $\mathrm{Ca}$ signaling with the novel concept of F-actin-based Ca signaling. Crit. Rev. Eukaryot. Gene Expr. 2006, 16, 307-365.

[52] O'Rourke, M. F., Arterial aging: pathophysiological principles. Vasc. Med. 2007, 12, 329-341.

[53] Cooper, G. J., Young, A. A., Gamble, G. D., Occleshaw, C. J. et al., A copper(II)-selective chelator ameliorates leftventricular hypertrophy in type 2 diabetic patients: a randomised placebo-controlled study. Diabetologia 2009, $52,715-722$.

[54] Gong, D., Chen, X., Middleditch, M., Huang, L. et al., Quantitative proteomic profiling identifies new renal targets of copper(II)-selective chelation in the reversal of diabetic nephropathy in rats. Proteomics 2009, 9, $4309-4320$ 